supporting maternal mental health in pregnancy are important to address the early emergence of inequalities in child mental health. Parent-reported child mental health, and cohort attrition are limitations of this study.

\section{OP22 UNDERSTANDING SOCIAL INEQUALITIES IN BEING BULLIED IN CHILDHOOD: FINDINGS FROM THE UK MILLENNIUM COHORT STUDY}

M Campbell* ${ }^{*}$ V Straatmann, D Taylor-Robinson. Department of Public Health and Policy, University of Liverpool, Liverpool, UK

10.1136/jech-2017-SSMAbstracts.22

Background Childhood bullying is an important policy concern. Nearly half of victims of bullying report thoughts about suicide and self-harm with negative impacts extending across the life-course. Being bullied in childhood is common, socially patterned, however, factors explaining social inequalities in being bullied are unclear. Using a contemporary United Kingdom (U.K.) birth cohort, we aimed to assess and explain social inequalities in the risk of being bullied.

Methods Analysis of the U.K. Millennium Cohort Study using a sample of 12706 children surveyed at four sweeps (aged nine months, three, five and seven years). The main outcome was a binary, child-reported measure of being bullied at age 7 . Household income quintile at birth was the main measure of socio-economic conditions. Relative risk (RR) and 95\% confidence intervals $(95 \% \mathrm{CI})$ for being bullied were estimated using Poisson regression, by household income quintile. Sequential models adjusted for risk factors for being bullied, including individual (e.g. emotional resilience, health status including obesity), parental (e.g. maternal mental health and discipline) and peer relationship (e.g. friends) factors. Analysis used Stata/SE with svy commands to account for the sampling design and attrition. Our sensitivity analysis will use parent and teacher reported outcome measures.

Results By age seven, 48.7\% (95\%CI 47.5\%-49.9\% $[\mathrm{n}=6183])$ of children self-reported being bullied. There was a social gradient; $53.4 \%$ (95\%CI 50.6\%-56.1\%) in the lowest income quintile reported having been bullied, compared to 43.9\% (95\%CI 41.5\%-46.4\%) in the highest (RR 1.21 [95\% CI 1.10-1.33]). Male sex, young maternal age, higher child BMI and Strengths and Difficulties Questionnaire (SDQ) scores and worse maternal mental health were independently associated with an increased risk of being bullied, whilst having a good friend was protective. Controlling for these factors in the final model attenuated the RR for being bullied for children in the lowest income quintile, compared to the highest, rendering the association non-significant (RR 1.08 [95\%CI 0.98-1.19]).

Conclusion Using a nationally representative cohort, about half of seven year olds reported being bullied, with a greater risk in children from the poorest homes. Increased risk was largely explained by social differences in other risk factors for bullying including friends, maternal mental health, and individual factors including emotional resilience and BMI. Interventions addressing these risks and promoting protective factors are likely to reduce social inequalities in being bullied, improving mental health outcomes for the most vulnerable U.K. children. Future research should investigate inequalities in being bullied in adolescents. The self-reported primary outcome is the main study limitation.

\section{OP23 MODELLING THE POTENTIAL FOR PARENTING INTERVENTIONS TO REDUCE INEQUALITIES AND POPULATION PREVALENCE OF CHILDREN'S MENTAL HEALTH PROBLEMS: EVIDENCE FROM THE MILLENNIUM COHORT STUDY (MCS)}

${ }^{1} \mathrm{~S}$ Hope $*{ }^{1} \mathrm{~A}$ Pearce, ${ }^{1} \mathrm{M}$ Cortina-Borja, ${ }^{2} \mathrm{C}$ Chittleborough, ${ }^{1} \mathrm{C}$ Law. ${ }^{1}$ Population, Policy and Practice Programme, UCL Great Ormond Street Institute of Child Health, London, UK; ${ }^{2}$ School of Public Health, The University of Adelaide, Adelaide, Australia

\subsection{6/jech-2017-SSMAbstracts.23}

Background Parenting programmes aim to support parents' skills and confidence, improving parenting and, in turn, children's mental health. Thus they have the potential to reduce population prevalence (and inequalities) in child mental health problems. We modelled the potential population impact of scale-up of parenting interventions in a national cohort. Based on review evidence, we simulated interventions with effect sizes of 0.9SD (intensive) and 0.4SD (standard).

Methods We used data from the UK Millennium Cohort Study, following 18000 children born 2000-2002. Parenting was assessed by Child-Parent Relationship Scale (CPRS: ShortForm) score when the child was 3 years. Child mental health problems (CMHP) at 5 years were assessed by Strengths and Difficulties Questionnaire (SDQ) total score, dichotomised using an established cut-off. Socio-economic circumstances were represented by mother's highest academic qualifications (<GCSEs A-C; GCSEs A-C and above) at 9 months.

Predicted probabilities of CMHP were estimated, fitting marginal structural models to examine the mediating effect of parenting, accounting for confounding using inverse-probability-treatment-weights. Inequalities were assessed with Risk Ratios (RR [95\% CIs]). A series of intervention scenarios were simulated by re-estimating predicted probabilities after modifying the CPRS score (to reflect effectiveness) for eligible children (targeting).

We analysed data from 14540 children, using Stata 13.1. Survey weights and multiple imputation addressed missing data.

Results Overall prevalence of CMHP at 5 years was $10.8 \%$, and children of mothers with low educational attainment had a greater risk of CMHP $(\mathrm{RR}=2.46 \quad$ [95\%CI:2.24-2.70]). Focusing on three parenting intervention scenarios, we showed changes in CMHP prevalence and inequalities compared to those observed: 1. Universal (standard support to all parents): prevalence change $-2.5 \%$; inequality $R R=2.56[2.30-2.85] ; 2$. Targeted (intensive support to families receiving means-tested benefits): prevalence change $-1.4 \%$; inequality $\mathrm{RR}=2.11$ [1.91-2.34]; 3. Progressive universal (intensive support for families receiving benefits and standard support for others): prevalence change $-3.2 \%$; inequality $\mathrm{RR}=2.35$ [2.10-2.62].

Conclusion Large inequalities in CMHP were apparent by age 5 years. In simulated scenarios, inequality was reduced through an intervention that explicitly set out to provide intensive support to disadvantaged families. In contrast, reductions in overall prevalence were more likely to be achieved by universal interventions. A progressive universal approach (combining intensive support for disadvantaged families with standard support for others) led to a reduction in both population prevalence and inequality. Nevertheless, in all intervention scenarios, inequalities in CMHP remained strong. These results suggest that parenting interventions may contribute to a reduction in CMHP inequalities, particularly when including targeted support for disadvantaged families. 